\title{
Hopfield networks: from optimization to adaptive control $^{*}$
}

\author{
Miguel Atencia \\ Departamento de Matemática Aplicada \\ Campus de Excelencia Internacional Andalucía TECH \\ Universidad de Málaga \\ Email: matencia@ctima.uma.es
}

\author{
Gonzalo Joya \\ Departamento de Tecnología Electrónica \\ Email: gjoya@uma.es
}

\begin{abstract}
This paper proposes an adaptive control algorithm, which is designed by adding a parametric identification method to a non-linear controller. The identification module is built upon the Hopfield neural network, resulting in an unconventional network with time-varying weights and biases. The convergence of the estimations of the parameters of a dynamical system was proved in previous work, as long as the system inputs can be freely manipulated to provide persistent excitation. Henceforth the behaviour of the closed-loop system, when the inputs result from the controller equations, is here analyzed in order to assess both the tracking performance of the full adaptive controller and the identification ability of the neural estimator. The algorithm is applied to an idealized robotic system with two joints, whose positions and velocities are required to follow, as closely as possible, a prescribed reference trajectory. The simulation results show a satisfactory control performance, since the demanded trajectory is almost accurately followed. The estimated values also converge to the correct parameters, as long as the controller provides sufficiently rich signals to the system. The results are similar to a conventional least-squares adaptive controller, with a much lower computational cost.
\end{abstract}

\footnotetext{
* Preprint
} 